\title{
Confirmation of gestational age by external physical characteristics (total maturity score)
}

\author{
JOHN I. CATER \\ Department of Child Health, Ninewells Hospital and Medical School, Dundee
}

\begin{abstract}
SUMMARY The use of the total maturity score (TMS) as a means of estimating gestational age was assessed in a population of 76 low birthweight infants $(\leq 2500$ g) and 80 matched controls $(>2500 \mathrm{~g})$ all of certain gestational ages. The gestational ages, derived from the last menstrual period and from the TMS, were similar except in preterm low birthweight infants. The discrepancy in this group was attributed to the structure of the scoring system. It is concluded that the TMS is a convenient and accurate method of assessing gestational age in term babies.
\end{abstract}

Considerable research has recently been devoted to evolving methods of corroborating gestational age as calculated from the date of the last menstrual period (LMP). These range from the use of ultrasound, $x$-rays, and amniotic fluid estimations during pregnancy, to biochemical and clinical procedures in the newborn. The last method can be divided into three main groups-biochemical, anthropometric, and nonanthropometric. The most widely used are external physical characteristics (Farr et al., 1966), neurological signs (Robinson, 1966), or both (Dubowitz et al., 1970).

The purpose of the study reported in this paper was to assess the use of the total maturity score (TMS) (Farr et al., 1966) in a total low birthweight (LBW) population as a means of estimating gestational age as derived from the date of the LMP. Particular attention was paid to its accuracy in preterm LBW infants.

\section{Method}

The infants studied were a sample from a larger survey of low birthweight babies (Cater, 1978) and comprised LBW infants $\leq 2500 \mathrm{~g}$ and their matched controls of normal birthweights $(>2500 \mathrm{~g})$. All were of certain gestational ages and each infant was examined by J.I.C. It was fundamental to the study that J.I.C. should not know the length of gestation as stated by the mother and that the maternal history be assessed by an obstetrician, thus ensuring an independent decision concerning the accuracy of the maternal data.

The method recommends that the baby should be examined between 12 and 36 hours of age. The mean age for examination of the controls was $22 \cdot 1 \pm 7 \cdot 6$ hours; total LBW $23 \cdot 2 \pm 13 \cdot 1$ hours; LBW $24 \cdot 8$ $\pm 17 \cdot 6$ hours; and term LBW $21 \cdot 7 \pm 7 \cdot 0$ hours. There was no significant difference between the mean age at examination of preterm LBW infants and controls. The preterm infants were examined at a later age because of the higher incidence of illness in them.

\section{Results}

The data are presented and analysed in broad categories of controls and total LBW, with more detailed subdivision into preterm LBW and term LBW to reveal any weakness of the method. In other studies the results have been combined and presented for a single group, thus concealing the difficulties posed by the preterm subgroup.

The gestational age was derived from both the LMP and the TMS and the difference between the two methods was compared (Table). The only significant difference was found in the preterm LBW group in which the mean gestational age derived from the LMP, $32 \cdot 9$ weeks SD $2 \cdot 9$, differed from that derived from the TMS, $34 \cdot 4$ weeks SD $3 \cdot 0$ $(\mathrm{P}<0.05)$, a difference of 1.5 weeks. Comparison of the mean gestational ages derived from the LMP and TMS for the other three groups showed no statistical differences. The wide SD found in the total LBW group reflects the influenee of the preterm LBW group.

Table Comparison of the mean gestational ages in weeks calculated from the last menstrual period (LMP) and from the total maturity scores (TMS) in babies whose mothers were certain of their dates

\begin{tabular}{|c|c|c|c|c|c|c|c|c|}
\hline & \multicolumn{2}{|c|}{ Controls $(n=80)$} & \multicolumn{2}{|c|}{ Total LBW $(n=76)$} & \multicolumn{2}{|c|}{ Preterm LBW $(n=36)$} & \multicolumn{2}{|c|}{ Term LBW $(n=40)$} \\
\hline & Mean & $S D$ & Mean & $S D$ & Mean & $S D$ & Mean & $S D$ \\
\hline $\begin{array}{l}\text { Gestational age } \\
\text { Derived from LMP } \\
\text { Derived from TMS }\end{array}$ & $\begin{array}{l}40 \cdot 1 \\
40 \cdot 4\end{array}$ & $\begin{array}{l}1.0 \\
1.0\end{array}$ & $\begin{array}{l}36 \cdot 1 \\
36 \cdot 6\end{array}$ & $\begin{array}{l}3 \cdot 2 \\
3 \cdot 2\end{array}$ & $\begin{array}{l}32 \cdot 9^{*} \\
34 \cdot 4^{*}\end{array}$ & $\begin{array}{l}2.6 \\
3.0\end{array}$ & $\begin{array}{l}39.0 \\
38.6\end{array}$ & $\begin{array}{l}1.1 \\
1.8\end{array}$ \\
\hline
\end{tabular}

$\rightarrow \mathrm{P}<0.05$ 


\section{Discussion}

External physical characteristics as a means of assessing gestational age were preferred to neurological signs for two reasons. Firstly, the sample was biased towards LBW, and therefore many infants would be attached to intensive care equipment, making impossible a number of tests needed for the neurological examination; secondly, it was felt that neurological signs might be altered by the delivery process and drugs given to the mother.

A number of methodological problems are evident in earlier studies. Prior knowledge of maternal dates has been a serious difficulty in several, where the investigator has both examined the baby and questioned the mother concerning the details of the LMP. Such a dual role inevitably influences the findings: the maternal menstrual data must be assessed by an independent worker.

The use of a correlation coefficient has been common in previous studies but has not been entirely satisfactory due to the paucity of babies of shorter gestations. In the present sample a relatively large number of preterm babies of known gestational ages $(36 / 156)$ was included. For this reason the sample was subdivided into preterm and term LBW groups and analysed separately. The analysis highlights the technical problems of the score. It has not always been appreciated by previous investigators that the precision of the TMS varies with gestational age, since 0.9 points represents a week before 30 weeks and 0.8 points equals a week before 32 weeks, whereas at 39 weeks, 3 points represent a week. The difference in the mean gestational age for preterm infants calculated by the TMS method from that derived from the LMP is 1.5 weeks, compared with 0.4 weeks for infants at term, which shows the inaccuracy of the use of this score in the preterm LBW group

The use of external characteristics seems an easy and satisfactory method of assessing the gestational age of the term baby, and the error is well within the range acceptable in other analyses using complex biological investigations. The results for term infants are remarkably consistent, considering the vagaries of maternal memory and the tacit assumption that ovulation occurs on the 14th day of each cycle. In contrast, the results obtained for preterm infants underline the inaccuracies and uncertainty present in methods currently available for corroborating the maternal data in this group. The small numbers of infants, the effects of illness and drugs, type of delivery, and the structure of the score all militate against accurate assessment in these infants, and further study of this problem is needed.

\section{Conclusion}

For routine clinical purposes the use of the external physical characteristics is a simple and convenient method for estimating gestational age. It is particularly accurate at term but is less precise for babies of short gestation.

I thank Professor John Dennis for reviewing the maternal data, Mrs Phyllis Easton for technical assistance, Professor R. G. Mitchell for his helpful comments, and Action for the Crippled Child for generous financial assistance.

\section{References}

Cater, J. (1978). A population study of LBW infants and their matched controls with special reference to fetal growth. MD thesis, University of Edinburgh.

Dubowitz, L. M. S., Dubowitz, V., and Goldberg, C. (1970). Clinical assessment of gestational age in the newborn infant. Journal of Pediatrics, 77, 1-10.

Farr, V., Kerridge, D. F., and Mitchell, R. G. (1966). The value of some external characteristics in the assessment of gestational age at birth. Developmental Medicine and Child Neurology, 8, 657-660.

Robinson, R. J. (1966). Assessment of gestational age by neurological examination. Archives of Disease in Childhood, 41, 437-447.

Correspondence to Dr John I. Cater, Department of Child Health, Ninewells Hospital and Medical School, Dundee DD1 9SY, Angus.

\section{Dissolution of bilateral staghorn cystine renal calculi}

\section{G. RUYSCH VAN DUGTEREN AND J. WIGGELINKHUIZEN \\ Department of Paediatrics, University of Cape Town, and Renal Clinic, Red Cross War Memorial Children's Hospital, Cape Town}

SUMMARY Bilateral staghorn renal calculi in a 7year-old girl with cystinuria were dissolved over a period of 6 months, using a high fluid intake, urinary alkalinisation, and D-penicillamine. Even in children with extensive cystine urolithiasis, medical management may avert the need for surgery.
Cystinuria is an inherited complex autosomal recessive defect in which there is decreased proximal renal tubular reabsorption of filtered cystine, lysine, arginine, and ornithine. Defective transport of these amino-acids in the gut has also been demonstrated, but the clinical symptoms are related only to the associated stone formation (Crawhall and Watts, 